\title{
Yoga has Beneficial Effects on Patients with Chronic Diseases and Improves Immune Functions
}

\section{Nobuhiko Eda*}

Waseda University, Faculty of Sport Sciences, Tokorozawa, Saitama, Japan

${ }^{*}$ Corresponding author: Nobuhiko Eda, Waseda University, Faculty of Sport Sciences, Tokorozawa, Saitama, Japan, Tel: +81 429476721 ; Fax: +81 4 2947 6721; Email: e-nobuhiko@asagi.waseda.jp

Rec date: Jul 22, 2014, Acc date: Oct 16, 2014, Pub date: Oct 18, 2014

Copyright: ( 2014 Eda N, et al. This is an open-access article distributed under the terms of the Creative Commons Attribution License, which permits unrestricted use, distribution, and reproduction in any medium, provided the original author and source are credited.

\begin{abstract}
The practice of various styles of yoga has become popular in Western countries. Recently, chronic diseases, such as chronic obstructive pulmonary disease (COPD), cancer, cardiovascular diseases (CVD), obesity and diabetes mellitus, have emerged as serious problems worldwide, and it has become important for older adults to maintain or improve their immune functions. Yoga has been reported to have positive effects on distress and functional performance in patients with chronic diseases and improves several immunological indicators. In this paper, we review recent studies that have examined the effects of yoga on chronic diseases, especially COPD and cancer, and immune functions including those mediated by human $\beta$-defensin 2 (HBD-2), natural killer (NK) cells, and proinflammatory cytokines. We suggest that yoga has a potential use as a complementary and alternative therapy for chronic diseases, and we expect yoga to be useful for older adults who wish to maintain their health.
\end{abstract}

Keywords: Yoga; Chronic obstructive pulmonary disease; Cancer; Defensin; Natural killer cells; Proinflammatory cytokines

\section{Introduction}

The word "yoga" comes from Sanskrit "Yuj", which means to yoke or to join together. Yoga is thought to have originated in India approximately 4,500 years ago and several styles of yoga continue to be practiced in the world today. Raja yoga mainly involves mediation, and the eight phases of this yoga practice have considerably influenced other yoga styles. Hatha yoga, which emphasizes the notion of life energy (prana), is a driving force for the mind and body; practitioners of Hatha yoga attempt to control the mind by using the flow of prana. Ashtanga and Iyengar yoga are subtypes of Hatha yoga. Practitioners of Ashtanga yoga try to achieve a deeper state of meditation by harmonizing breathing with movements, whereas the purpose of Iyengar yoga is to take control of the mind by using postural regulation. The detailed adjustment of postures is the most significant feature of this yoga style. Recently, the popularity of various types of yoga in Western countries has increased, including power yoga that combines Ashtanga Iyengar styles, Bikram yoga that is practiced under hot and humid conditions, and laughter yoga that combines laughter exercises with yogic breathing. Recent studies in which the relationship between yoga and chronic diseases and immune functions has been examined are reviewed in this paper.

\section{Yoga and chronic diseases}

The World Health Organization has reported that 64 million people have developed chronic obstructive pulmonary disease (COPD). Three million people died of COPD in 2004, and it is predicted to become the third leading cause of death worldwide by 2030 [1]. COPD is a lung ailment characterized by persistent blockage of airflow and symptoms such as breathlessness, abnormal sputum, and chronic cough. In a study of 29 elderly patients with COPD, participation in a 12-week Iyengar yoga training program reduced dyspnea-related distress (Baseline; $2.6 \pm 2.8,12$-week; $1.6 \pm 1.7$, effect size $(E S)=0.67$, $\mathrm{p}=0.08$ ) and improved the functional performance of patients (Baseline; $2.0 \pm 0.5,12$-week; $2.2 \pm 0.4, \mathrm{ES}=0.79, \mathrm{p}=0.04$ ) compared to usual-care control group [2]. In another study of 25 patients with COPD, the anxiety score (pre-Iyengar yoga; $3.91 \pm 1.03$, post-Iyengar yoga; $4.50 \pm 0.76)$ in the Hospital Anxiety and Depression Scale and the fatigue score (pre-Iyengar yoga; $6.63 \pm 4.63$, post-Iyengar yoga; $3.25 \pm 1.98)$ in the Chronic Respiratory Questionnaire improved significantly. Moreover, the clinically important scores of these patients changed after they completed the 12-week Iyengar yoga program [3].

Cancer is a leading cause of death worldwide. According to a report released by the International Agency for Research on Cancer, the total number of cancer deaths was 8.2 million in 2012, with lung (1.59 million deaths), liver (745 thousand deaths), stomach (723 thousand deaths), colorectal (694 thousand deaths), and breast cancers (521 thousand deaths) causing the highest number deaths [4]. Chandwani et al. [5] investigated the effect of a 6-week yoga program on the quality of life (QOL) of 163 breast cancer patients. The physical component scale scores and physical functioning of the yoga group improved to a greater extent than those of the control group at 1 and 3 months after radiotherapy. In a study of mindfulness-based cancer recovery (MBCR), which involved mindfulness meditation and gentle yoga, stress symptoms and QOL showed greater improvements in the MBCR group than in the control group [6].

Cardiovascular diseases (CVD) are the most common cause of death in the world. According to an announcement by the World Health Organization (WHO), the number of deaths from CVD was about 17.3 million in 2008, representing $30 \%$ of all global deaths, and will increase to reach 23.3 million by 2030 [7-9]. The most important risk factors of CVD are obesity, diabetes mellitus. Sarvottam et al. [10] reported that a short-term yoga-based lifestyle intervention for 2 hours each day for 10 days decreased interleukin (IL)- 6 and increased adiponectin in 51 overweight and obese men. High levels of IL-6 and 
low levels of adiponectin were associated with CVD risk. Therefore, a short-term yoga-based lifestyle intervention may be an important modality to reduce the risk for CVD as indicated by a decrease in IL-6 and an increase in adiponectin in overweight and obese men. In the study of 231 patients with type 2 diabetes mellitus, fasting blood glucose, serum total cholesterol, very LDL and malondialdehyde decreased and the activity of superoxide dismutase increased in the yoga groups after the 6-months Hatha yoga program [11]. Hunter et al. [12] investigated the effect of Bikram yoga on arterial stiffness and insulin resistance in 24 young and 18 older adults. As a result, total and low-density lipoprotein cholesterol levels, plasma insulin concentrations, and scores on the homeostatic model of the assessment of insulin resistance decreased in older adults, and carotid artery compliance increased and $\beta$-stiffness index decreased in young adults after the yoga program.

Taken together, these finding suggest that yoga has positive effects on patients with chronic diseases including COPD, breast cancer, obesity and diabetes mellitus. The practice of various styles of yoga has become popular recently, and yoga has been used as complementary and alternative therapy in Western countries.

\section{Yoga and immune functions}

Recently, yoga has been reported to improve various immunological parameters. Defensin antimicrobial peptides exert their effects by destroying the hydrophobic core of the lipid bilayers belonging to microbes. Human $\beta$-defensin 2 (HBD-2) is expressed in the epithelial cells of the oral cavity and respiratory tract. In a previous study, we showed that yogic stretching for 90 min increased salivary HBD-2 concentrations and HBD-2 expression rate in 15 older adults [13]. HBD-2 expression is known to be responsive to emotional stress. Therefore, we proposed that the relaxing effect of yogic stretching decreases stress, in turn increasing salivary HBD-2 expression.

Natural killer (NK) cells, a type of lymphocyte, play an important part in the innate immune system by eliminating damaged and cancer cells. The low cytotoxic activity of NK cells is associated with increased cancer risk. Kochupillai et al. [14] investigated the effects of yogic breathing on the numbers NK cells in cancer patients. In the yogicbreathing group, the numbers of NK cells increased significantly above baseline levels after 12 and 24 weeks of the practice and, after 24 weeks, significantly more NK cells were observed in the yogicbreathing group than in the control group. Witek-Janusek et al. [15] examined the relationship between Mindfulness-based Stress Reduction (MBSR), including yogic breathing practices, and immune function. Ninety-six women with breast cancer participated in a MBSR program for 8 weeks. NK cell activity and cytokine levels in the MBSR group returned to levels that comparable to those of the normal cancer-free group 4 weeks after completing MBSR therapy. In contrast, breast cancer patients in the non-MBSR group had low levels of NK cell activity and interferon (IFN) $-\gamma$ production, and high levels of IL-4, IL-6, and IL-10 production. NK cells and T cells are the predominant cells types that secrete IFN- $\gamma$. IFN- $\gamma$ exerts its proinflammatory function by activating endothelial cells, macrophages, and $\mathrm{T}$ cells, and by promoting the differentiation of $\mathrm{T}$ helper 1 (Th1) cells. Therefore, a decrease in serum IFN- $\gamma$ levels indicates a decline in cellular immunity. Researchers have shown that serum IFN- $\gamma$ levels decrease in patients under examination stress, however, IFN- $\gamma$ levels in a group of patients who practiced yoga for 12 weeks were lower than in those belonging to the control group [16]. These findings suggest that the practice of yoga could inhibit the decline in cellular immunity that is associated with psychological stress.

Proinflammatory cytokines are known to modulate the pathologies of various chronic diseases and therapies for patients with chronic diseases are designed to reduce inflammation. The activations of transforming growth factor (TGF) $\beta$, nuclear factor (NF)- $\kappa \mathrm{B}$, tumor necrosis factor (TNF)- $\alpha$ and IL- 6 have been reported in the previous studies of COPD, chronic kidney disease, obesity and metabolic syndrome [17-19]. Yadav et al. [20] reported that yoga reduced stress and inflammation makers in 86 patients with chronic inflammatory diseases who participated in a short-term yoga-based lifestyle program for 10 days. The mean level of cortisol decreased and $\beta$-endorphins increased from baseline by day 10 in these patients. Mean reductions in IL- 6 and TNF- $\alpha$ levels from baseline was also observed by day 10. In a study in which breast cancer patients participated in a 12 -week Iyengar yoga program, the plasma levels of soluble TNF receptor type II, a marker of TNF activity, remained stable in the yoga group but increased in the control group [21]. In another study, 12-week Hatha yoga intervention improved the vitality of breast cancer survivors, while reducing the fatigue score estimated by the Multidimensional Fatigue Symptom Inventory - Short Form and the levels of IL-6, TNF$\alpha$, and IL-1 $\beta 3$ months post-treatment. Increasing yoga practice decreases IL- 6 and IL- $1 \beta$ production, but not TNF- $\alpha$ production [22]. Therefore, regular yoga exercise might reduce the levels of proinflammatory cytokines and could be an effective treatment for chronic diseases.

\section{Conclusion}

Here, we reviewed the effects of yoga practice on chronic diseases and immune functions. These results suggest that yoga might benefit patients with chronic diseases, decreasing their distress and improving their functional performance. The positive effects of yoga on several immunological indicators have been reported recently; thus, yoga could be effectively used by older adults to prevent infectious diseases. Yoga has potential utility as a complementary and alternative therapy for chronic diseases and can help older adults to maintain their health.

\section{Key message}

Yoga is a low- or moderate-intensity exercise that is practice for the purpose of relaxation, and it can be performed comfortably and easily by beginners and older adults. In this paper, we reviewed the beneficial effects of yoga on COPD, cancer, CVD, obesity, diabetes mellitus and immune functions; however, numerous studies have reported various positive effects of yoga on other diseases and mental stress. Therefore, yoga might be useful for the maintenance of health in older adults. A more detailed investigation of the effects of yoga on immune functions is warranted.

\section{References}

1. Martínez-Pérez B, de la Torre-Díez I, López-Coronado M (2013) Mobile health applications for the most prevalent conditions by the World Health Organization: review and analysis. J Med Internet Res 15: e120.

2. Donesky-Cuenco D, Nguyen HQ, Paul S, Carrieri-Kohlman V (2009) Yoga therapy decreases dyspnea-related distress and improves functional performance in people with chronic obstructive pulmonary disease: a pilot study. J Altern Complement Med 15: 225-234.

3. Santana MJ, S-Parrilla J, Mirus J, Loadman M, Lien DC, et al. (2013) An assessment of the effects of Iyengar yoga practice on the health-related 
Citation: Eda N (2014) Yoga has Beneficial Effects on Patients with Chronic Diseases and Improves Immune Functions. J Clinic Res Bioeth 5: 197. doi:10.4172/2155-9627.1000197

Page 3 of 3

quality of life of patients with chronic respiratory diseases: a pilot study. Can Respir J 20: e17-23.

4. Forsea AM, Del Marmol V, de Vries E, Bailey EE, Geller AC (2012) Melanoma incidence and mortality in Europe: new estimates, persistent disparities. Br J Dermatol 167: 1124-1130.

5. Chandwani KD, Perkins G, Nagendra HR, Raghuram NV, Spelman A, et al. (2014) Randomized, controlled trial of yoga in women with breast cancer undergoing radiotherapy. J Clin Oncol 32: 1058-1065.

6. Carlson LE, Doll R, Stephen J, Faris P, Tamagawa R, et al. (2013) Randomized controlled trial of Mindfulness-based cancer recovery versus supportive expressive group therapy for distressed survivors of breast cancer. J Clin Oncol 31:3119-3126.

7. World Health Organization (2013) Cardiovascular diseases (CVDs). Fact sheet $\mathrm{N}^{\circ} 317$.

8. World Health Organization (2011) Global status report on noncommunicable diseases 2010.

9. Mathers CD, Loncar D (2006) Projections of global mortality and burden of disease from 2002 to 2030. PLoS Med 3: e442.

10. Sarvottam K, Magan D, Yadav RK, Mehta N, Mahapatra SC (2013) Adiponectin, interleukin-6, and cardiovascular disease risk factors are modified by a short-term yoga-based lifestyle intervention in overweight and obese men. J Altern Complement Med 19: 397-402.

11. Gordon LA, Morrison EY, McGrowder DA, Young R, Fraser YT, et al. (2008) Effect of exercise therapy on lipid profile and oxidative stress indicators in patients with type 2 diabetes. BMC Complement Altern Med 8: 21.

12. Hunter SD, Dhindsa MS, Cunningham E, Tarumi T, Alkatan M, et al. (2013) The effect of Bikram yoga on arterial stiffness in young and older adults. J Altern Complement Med 19: 930-934.

13. Eda N, Shimizu K, Suzuki S, Tanabe Y, Lee E, et al. (2013) Effects of yoga exercise on salivary beta-defensin 2. Eur J Appl Physiol 113: 2621-2627.

14. Kochupillai V, Kumar P, Singh D, Aggarwal D, Bhardwaj N, et al. (2005) Effect of rhythmic breathing (Sudarshan Kriya and Pranayam) on immune functions and tobacco addiction. Ann N Y Acad Sci 1056: $242-252$.

15. Witek-Janusek L, Albuquerque K, Chroniak KR, Chroniak C, DurazoArvizu R, et al. (2008) Effect of mindfulness based stress reduction on immune function, quality of life and coping in women newly diagnosed with early stage breast cancer. Brain Behav Immun 22: 969-981.

16. Gopal A, Mondal S, Gandhi A, Arora S, Bhattacharjee J (2011) Effect of integrated yoga practices on immune responses in examination stress - A preliminary study. Int J Yoga 4: 26-32.

17. López-Hernández FJ, López-Novoa JM (2012) Role of TGF- $\beta$ in chronic kidney disease: an integration of tubular, glomerular and vascular effects. Cell Tissue Res 347: 141-154.

18. Edwards MR, Bartlett NW, Clarke D, Birrell M, Belvisi M, et al. (2009) Targeting the NF-kappaB pathway in asthma and chronic obstructive pulmonary disease. Pharmacol Ther 121: 1-13.

19. Khosravi R, Ka K, Huang T, Khalili S, Nguyen BH, et al. (2013) Tumor necrosis factor- $\alpha$ and interleukin-6: potential interorgan inflammatory mediators contributing to destructive periodontal disease in obesity or metabolic syndrome. Mediators Inflamm: 1-6.

20. Yadav RK, Magan D, Mehta N, Sharma R, Mahapatra SC (2012) Efficacy of a short-term yoga-based lifestyle intervention in reducing stress and inflammation: preliminary results. J Altern Complement Med 18: 662-667.

21. Bower JE, Greendale G, Crosswell AD, Garet D, Sternlieb B, et al. (2014) Yoga reduces inflammatory signaling in fatigued breast cancer survivors: a randomized controlled trial. Psychoneuroendocrinology 43: 20-29.

22. Kiecolt-Glaser JK, Bennett JM, Andridge R, Peng J, Shapiro CL, et al. (2014) Yoga's impact on inflammation, mood, and fatigue in breast cancer survivors: a randomized controlled trial. J Clin Oncol 32: 1040-1049. 